\title{
Intranasal administration of RSV antigen-expressing MCMV elicits robust tissue-resident effector and effector memory CD8 + T cells in the lung
}

\begin{abstract}
KM Morabito ${ }^{1,2}$, TR Ruckwardt ${ }^{1}$, AJ Redwood ${ }^{3}$, SM Moin ${ }^{1}$, DA Price ${ }^{4,5}$ and BS Graham ${ }^{1}$
Cytomegalovirus vectors are promising delivery vehicles for vaccine strategies that aim to elicit effector CD8 + Tcells. To determine how the route of immunization affects $C D 8+T$-cell responses in the lungs of mice vaccinated with a murine cytomegalovirus vector expressing the respiratory syncytial virus matrix (M) protein, we infected CB6F1 mice via the intranasal or intraperitoneal route and evaluated the M-specific CD8 + T-cell response at early and late time points. We found that intranasal vaccination generated robust and durable tissue-resident effector and effector memory CD8 + T-cell populations that were undetectable after intraperitoneal vaccination. The generation of these antigen-experienced cells by intranasal vaccination resulted in earlier T-cell responses, interferon gamma secretion, and viral clearance after respiratory syncytial virus challenge. Collectively, these findings validate a novel approach to vaccination that emphasizes the route of delivery as a key determinant of immune priming at the site of vulnerability.
\end{abstract}

\section{INTRODUCTION}

Tissue-resident memory $\mathrm{T}\left(\mathrm{T}_{\mathrm{RM}}\right)$ cells have been found at many barrier sites, including the skin, gastrointestinal tract, and lung, where they are poised to respond quickly to invading pathogens. ${ }^{1-4}$ Unlike effector memory $\mathrm{T}\left(\mathrm{T}_{\mathrm{EM}}\right)$ cells, which may recirculate, $\mathrm{T}_{\mathrm{RM}}$ cells remain in the tissue and are often identified by the expression of CD69, an early activation marker, and CD103, an $\alpha \mathrm{E}$ integrin, although the latter may vary at different anatomical locations. ${ }^{1,5} \mathrm{~T}_{\mathrm{RM}}$ cells act as antigen-specific sentinels and recruit innate and adaptive immune cells into the infected tissue via the secretion of cytokines and chemokines. ${ }^{6,7}$ They also proliferate rapidly and kill infected cells, protecting directly against local challenge. ${ }^{3,4,5,8}$ It may therefore be important to elicit $T_{R M}$ cells as part of an effective vaccination strategy.

Most respiratory virus infections are self-limited, and replication-defective vaccine vectors only express antigen for a short time. Immunization through brief exposure to antigen in these settings typically leads to the generation of central memory $\mathrm{T}\left(\mathrm{T}_{\mathrm{CM}}\right)$ cells, which are long-lived but slow to regain effector functions. ${ }^{9-11}$ In contrast, persistent vectors such as cytomegalovirus (CMV) produce antigen for longer periods of time and generate high frequencies of $\mathrm{T}_{\mathrm{EM}}$ cells, which respond quickly to subsequent infection. CMV-based vaccines have proven to be effective against simian immunodeficiency virus infection in Rhesus macaques. ${ }^{12}$ Although vaccination did not lead to sterilizing immunity, challenged animals were able to control viremia to undetectable levels. Protection was attributed to the generation of $\mathrm{CD} 4+$ and $\mathrm{CD} 8+\mathrm{T}_{\mathrm{EM}}$ cells, which were able to clear virus from peripheral tissues. ${ }^{13}$ This approach has also been shown to protect against other infectious diseases, including Ebola and tuberculosis, in various animal models. ${ }^{12,14-18}$ Similar vectors have been evaluated for therapeutic utility against cancer leading to delayed growth or rejection of tumors and even for the purpose of immunocontraception in mice. ${ }^{19-21}$

The phenomenon of memory inflation has been observed in murine CMV (MCMV) infection, and large populations

\footnotetext{
${ }^{1}$ Viral Pathogenesis Laboratory, Vaccine Research Center, National Institute of Allergy and Infectious Diseases, National Institutes of Health, Bethesda, Maryland, USA. ${ }^{2}$ Department of Microbiology and Immunology, Georgetown University Medical Center, Washington, District of Columbia, USA. ${ }^{3}$ Institute for Immunology and Infectious Diseases, Murdoch University, Perth, Western Australia, Australia. ${ }^{4}$ Institute of Infection and Immunity, Cardiff University School of Medicine, Cardiff, UK and ${ }^{5}$ Human Immunology Section, Vaccine Research Center, National Institute of Allergy and Infectious Diseases, National Institutes of Health, Bethesda, Maryland, USA. Correspondence: BS Graham (bgraham@mail.nih.gov)
} 
of CMV-specific memory $\mathrm{T}$ cells are found in elderly humans. ${ }^{22-26}$ During MCMV infection, some antigen-specific CD8 + T cells follow canonical memory kinetics, with an early expansion phase followed by rapid contraction after viral containment and the establishment of a low-level stable memory population. In contrast, other antigen-specific CD8 + T-cell populations undergo memory inflation and continue to accumulate throughout chronic infection. ${ }^{22,25,27}$ Inflationary $\mathrm{T}$ cells typically display an effector $\left(\mathrm{T}_{\mathrm{EFF}}\right)$ phenotype, with low expression of CD127 and CD62L and high expression of the terminal differentiation marker KLRG-1 (KLRG-1 $\left.+\mathrm{T}_{\mathrm{EFF}}\right)^{22,23,25,27-30}$ This unique phenomenon, which leads to sustained levels of functional MCMV-specific $\mathrm{CD} 8+\mathrm{T}$ cells, may provide an advantage for vaccination.

In this study, we investigated how the route of administration affects the generation of CD8 + T-cell responses following immunization with an MCMV vector expressing the respiratory syncytial virus (RSV) matrix (M) protein (MCMV-M). ${ }^{31,32}$ We show that intranasal (IN) vaccination with MCMV-M generates a robust and durable tissue-resident memory population with a $\mathrm{T}_{\mathrm{EFF}} / \mathrm{T}_{\mathrm{EM}}$ phenotype that is absent in mice vaccinated via the intraperitoneal (IP) route. Furthermore, tissue-resident memory $\mathrm{CD} 8+\mathrm{T}$ cells generated by IN vaccination respond rapidly upon antigen re-exposure, leading to lower viral loads after RSV challenge.

\section{RESULTS \\ Vaccination with MCMV-M induces an inflationary M-specific CD8 + T-cell response}

We first evaluated whether vaccination with a recombinant MCMV vector expressing the RSV M protein could generate an $\mathrm{M}$-specific CD8 + T-cell response in the lungs. CB6F1 mice were infected with RSV IN or vaccinated with MCMV-M IN or IP. The percentage of CD8 + T cells specific for the $D^{b} M_{187-195}$ epitope (M-specific) was determined using tetramer staining and flow cytometry at week 1 and week 6 post-infection in the lungs. Mice infected with RSV showed a peak response of $6.25 \%$ (Figure 1), which decreased after 6 weeks to $0.84 \%(P<0.01)$, consistent with memory contraction. In contrast, mice infected with MCMV-M mounted an M-specific CD8 + T-cell response that increased between week 1 and week 6 postinfection, consistent with MCMV-driven memory inflation. ${ }^{27}$ At 6 weeks post-infection, mice vaccinated IN with MCMV-M had significantly more $\mathrm{M}$-specific CD8 $+\mathrm{T}$ cells in the lungs compared with mice infected with $\mathrm{RSV}(P<0.0001)$. Interestingly, despite an identical dose of MCMV-M, mice vaccinated IN also had substantially higher frequencies of M-specific CD8 $+\mathrm{T}$ cells in the lungs at the same time point compared with mice vaccinated IP $(22.72 \%$ vs. $1.98 \%$, $P<0.0001)$.

\section{IN vaccination generates more $M$-specific CD8 $+\mathrm{T}$ cells in the lung parenchyma than IP vaccination}

To determine whether IN vaccination generates more tissuetropic M-specific CD8 $+\mathrm{T}$ cells in the lungs, we used an intravascular staining method that exclusively labels leukocytes

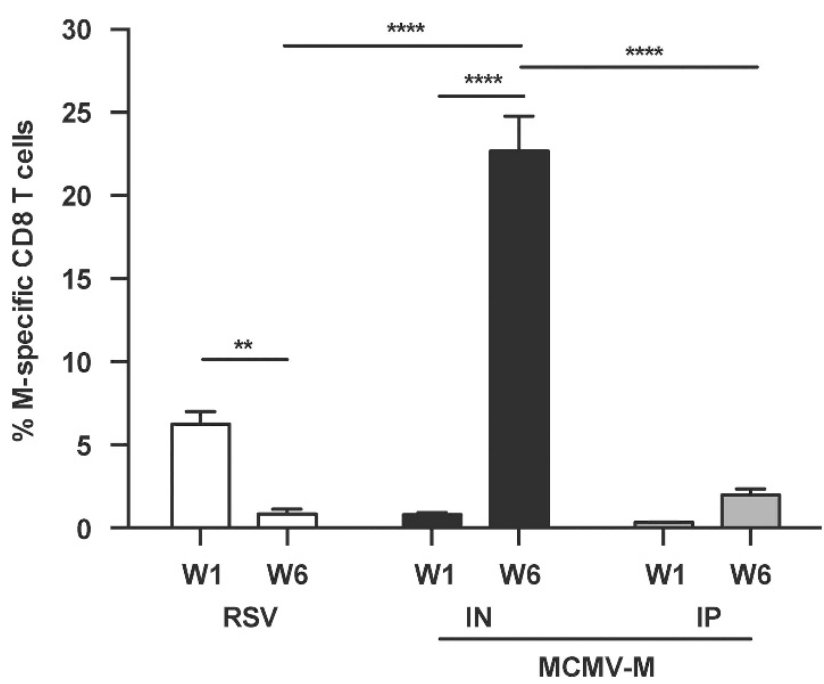

Figure 1 MCMV-M generates an M-specific CD8 + T-cell population that inflates over time. Mice were infected with $2 \times 10^{6} \mathrm{PFU}$ of RSV via the intranasal (IN) route or $6 \times 10^{5} \mathrm{PFU}$ of MCMV-M via the IN or intraperitoneal (IP) route. At week 1 (W1) and week 6 (W6) post-infection, the percentage of M-specific CD8 + T cells in the lungs was determined using tetramer staining and flow cytometry. Bars represent mean \pm s.e.m. with five mice per group. ${ }^{* * *} P \leq 0.0001$ and ${ }^{* *} P<0.01$ by two-way analysis of variance with Tukey's post-test for multiple comparisons. Data represent two independent experiments. MCMV-M, murine cytomegalovirus vector expressing the respiratory syncytial virus matrix (M) protein; RSV, respiratory syncytial virus.

in the blood, allowing us to distinguish between compartments. ${ }^{33}$ Mice were vaccinated IN or IP with MCMV-M, and $\mathrm{CD} 8+\mathrm{T}$-cell responses were evaluated in the lungs and spleen at weeks $1,6,16$, and 24 post-vaccination. Five minutes prior to killing, mice were injected intravenously with $3 \mu \mathrm{g}$ of fluorochrome-labeled anti-CD45 antibody. Cells that stained CD45IV + were located in the blood and available for immediate labeling following IV antibody administration, and cells that were CD45IV - were protected from IV antibody labeling and categorized as being in the tissue. As expected, IN administration of MCMV-M induced significantly greater frequencies and absolute numbers of $\mathrm{M}$-specific $\mathrm{CD} 8+\mathrm{T}$ cells in the lung parenchyma at weeks 6,16 , and 24 post-vaccination compared with an equivalent dose of MCMV-M administered IP $(P<0.0001$; Figure 2a and Supplementary Figure S1a online). However, there were no significant differences in the percentages or numbers of M-specific CD8 $+\mathrm{T}$ cells in the blood between vaccination groups. In the IN group, M-specific CD8 + T-cell frequencies in the blood and tissue increased between week 1 and week 6 post-vaccination, but significance was only achieved in the lung parenchyma $(P<0.0001)$. Similarly, the numbers of M-specific CD8 + T cells in the lung tissue inflated significantly between week 1 and week 6 postvaccination $(P<0.01$; see Supplementary Figure S1a). As a consequence, more $\mathrm{M}$-specific CD8 $+\mathrm{T}$ cells were present in the tissue compared with the blood of mice in the IN group at weeks 6,16 , and 24 post-vaccination $(P<0.01)$. In the IP group, $\mathrm{M}$-specific CD8 + T-cell numbers increased between week 1 and week 6 post-vaccination in the blood but not in the tissue 

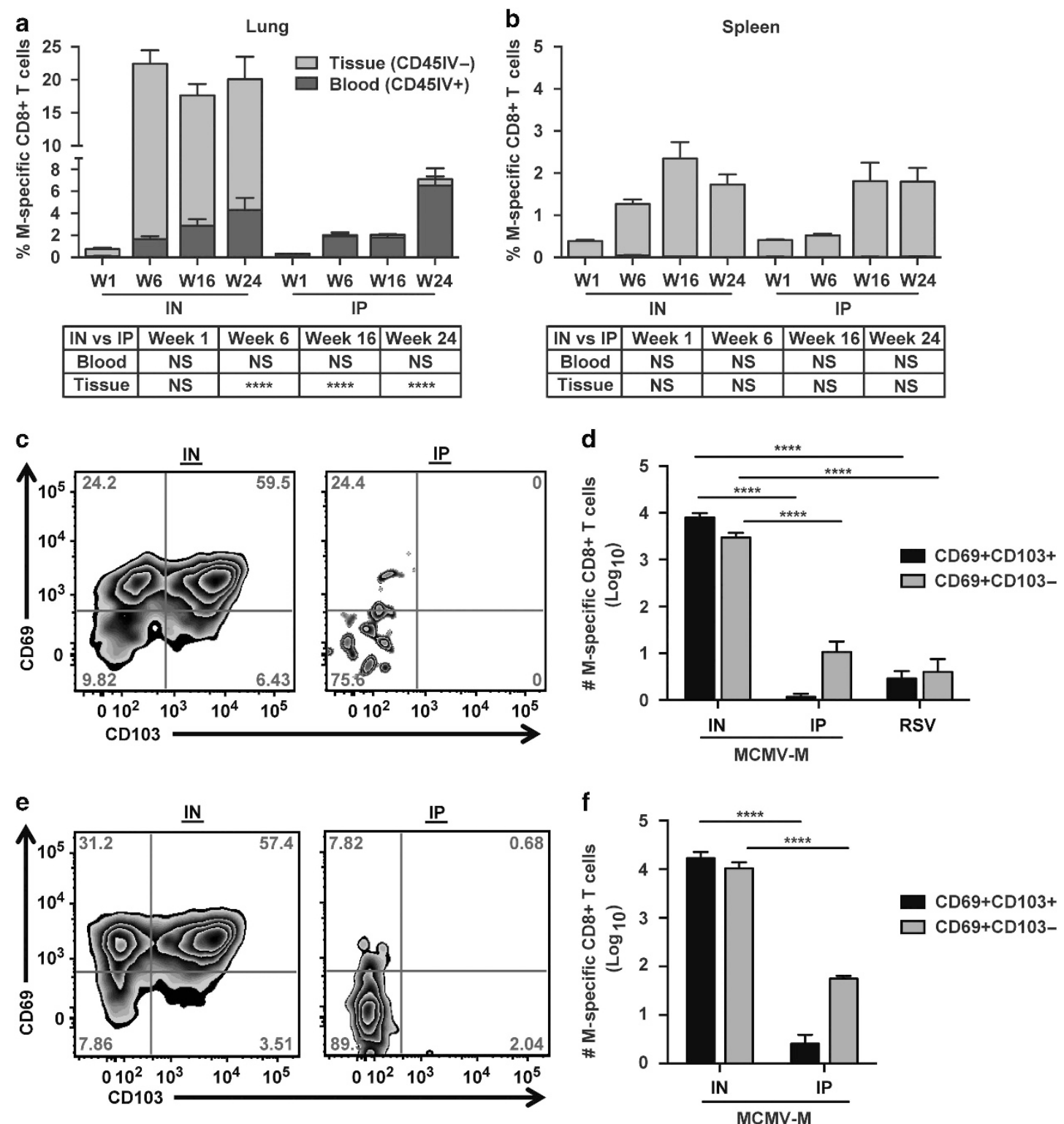

Figure 2 IN vaccination generates more M-specific CD8 + T cells in the lung parenchyma than IP vaccination. At weeks 1 (W1), 6 (W6), 16 (W16), and 24 (W24) post-vaccination with MCMV-M via the IN or IP route, mice were injected IV with anti-CD45 antibody 5 min prior to killing to identify cells in the blood (black) or tissue (gray). M-specific CD8 + T cells were identified by tetramer staining and flow cytometry. (a,b) Percentage of M-specific CD8 + T cells in the tissue and blood of the lungs in a and spleen in b. (c) Expression of CD69 and CD103 on M-specific CD8 + T cells in the lung tissue at 16 weeks post-vaccination. The percentage of CD8 + T cells in each quadrant is indicated. (d) Total number of CD69+CD103+ and CD69 + CD103 M-specific CD8 + T cells in the lung tissue at 16 weeks post-vaccination with MCMV-M or infection with RSV. (e) Expression of CD69 and CD103 on $\mathrm{M}$-specific CD8 + T cells in the lung tissue at 24 weeks post-vaccination. (f) Total number of CD69 + CD103 + and CD69+CD103 - M-specific CD8 + T cells in the lung tissue at 24 weeks post-vaccination with MCMV-M. Bars represent mean \pm s.e.m. with five mice per group. ${ }^{* * * *} P \leq 0.0001$ by two-way analysis of variance with Tukey's post-test for multiple comparisons. Data represent two independent experiments. IN, intranasal; IP, intraperitoneal; MCMV-M, murine cytomegalovirus vector expressing the respiratory syncytial virus matrix (M) protein; RSV, respiratory syncytial virus.

$(P<0.001$; see Supplementary Figure S1a). The frequencies of $\mathrm{M}$-specific CD8 $+\mathrm{T}$ cells in the spleen were not significantly different between the IN and IP vaccination groups at any time point (Figure $\mathbf{2 b}$ ). In both groups, significant inflation of the $\mathrm{M}$-specific CD8 + T-cell population in the spleen was apparent from week 1 to week 16 post-vaccination $(P<0.01)$. These data demonstrate that IN inoculation with MCMV-M establishes a robust $\mathrm{M}$-specific $\mathrm{CD} 8+\mathrm{T}$-cell population in the lung parenchyma that is not generated by IP vaccination. In contrast, equivalent systemic responses are induced in the blood and spleen irrespective of the mode of delivery, indicating that the increase in local tissue responses by IN vaccination is not at the expense of systemic immunity.

\section{IN vaccination with MCMV-M induces a tissue-resident memory M-specific CD8 + T-cell population}

Next, we determined whether the induced tissue-tropic $\mathrm{CD} 8+\mathrm{T}$ cells expressed CD69, an early activation marker 
typical of $\mathrm{T}_{\mathrm{RM}}$ cells, and $\mathrm{CD} 103$, an $\alpha \mathrm{E}$ integrin commonly used to delineate homing potential. At weeks 16 and 24 post-vaccination with MCMV-M via the IN or IP route, we evaluated the expression of these markers on M-specific $\mathrm{CD} 8+\mathrm{T}$ cells identified within the lung parenchyma on the basis of protection from intravascular staining. In the IN group, $61.8 \%$ of M-specific CD8 $+\mathrm{T}$ cells in the lung parenchyma expressed both CD69 and CD103, while only $0.86 \%$ of $\mathrm{M}$-specific CD8 $+\mathrm{T}$ cells expressed both markers in the IP group $(P<0.0001$; Figure 2c). This difference in the percentage of $\mathrm{T}_{\mathrm{RM}}$ was maintained through week 24 post-vaccination (Figure 2e; $P<0.0001$ ). We then determined the number of M-specific CD8 + T cells expressing CD69 and CD103 in the lung parenchyma in mice vaccinated IN or IP with MCMV-M and mice infected with RSV. As CD103 is not ubiquitously expressed on $\mathrm{T}_{\mathrm{RM}}$ cells, we also evaluated $\mathrm{CD} 69$ in isolation. We found approximately 1,000-fold more CD69+CD103 + and 100-fold more CD69 + CD103 - M-specific CD8 + T cells in the lung parenchyma of the IN group compared with the IP group $(P<0.0001$, Figure $2 \mathrm{~d})$. Moreover, IN vaccination with MCMV-M generated significantly greater numbers of $\mathrm{CD} 69+\mathrm{CD} 103+$ and CD69 + CD103 - M-specific CD8 + $\mathrm{T}$ cells compared with RSV infection $(P<0.0001$; Figure $2 \mathrm{~d})$. The number of $\mathrm{T}_{\mathrm{RM}}$ generated by IN vaccination with MCMV$M$ was maintained through week 24 post-vaccination (Figure 2f). Similar numbers of these tissue-resident subsets were present after RSV infection and IP vaccination with MCMV-M. These data show that M-specific CD8 $+\mathrm{T}_{\mathrm{RM}}$ cells in the lung are induced by vaccination with MCMV-M delivered IN but not IP.

\section{IN vaccination with MCMV-M augments the M-specific CD8 + T-cell response via the induction of effector and effector memory cells in the lung parenchyma and blood} Inflationary memory cells elicited by MCMV are comprised predominantly of KLRG-1 $+\mathrm{T}_{\mathrm{EFF}}$ cells, together with a smaller subset of $\mathrm{T}_{\mathrm{EM}}$ cells. We therefore evaluated the memory phenotype of M-specific CD8 $+\mathrm{T}$ cells in the tissue and blood of mice vaccinated IN or IP with MCMV-M. At weeks 1, 6, 16, and 24 post-vaccination, $\mathrm{M}$-specific $\mathrm{CD} 8+\mathrm{T}$ cells were identified by tetramer labeling after intravascular staining and subsequent tissue harvest. Expression of CD44, CD127, KLRG1, and CD62L were determined using flow cytometry. We categorized the M-specific CD8 + T cells into phenotypic subsets as follows: all, CD44+; central memory (CM), $\mathrm{CD} 127+\mathrm{KLRG} 1-\mathrm{CD} 62 \mathrm{~L}+$; effector memory (EM), $\mathrm{CD} 127+\mathrm{KLRG} 1-\mathrm{CD} 62 \mathrm{~L}-$; effector (E), CD127 KLRG1 - CD62L - ; and KLRG1 + effector (KLRG1 +), CD127 - CD62L - KLRG1 +. Overall, IN vaccination generated more $\mathrm{T}_{\mathrm{EFF}}$ and $\mathrm{T}_{\mathrm{EM}} \mathrm{M}$-specific $\mathrm{CD} 8+\mathrm{T}$ cells compared with IP vaccination at weeks 1 and $6(P<0.0001$; Figure 3a, b) and weeks 16 and $24 \quad(P<0.01$, see Supplementary Figure S2). Similar numbers of $\mathrm{T}_{\mathrm{CM}}$ and $\mathrm{KLRG} 1+\mathrm{T}_{\mathrm{EFF}}$ cells were present at weeks 6,16 , and 24. In the tissue, IN vaccination generated more $\mathrm{M}$-specific $\mathrm{CD} 8+$ T cells of all phenotypes at week 1 through week $16(P<0.05)$.
Although similar numbers of each phenotypic subset were present in the blood at week 1 post-vaccination, there were significantly more $\mathrm{T}_{\mathrm{EFF}}$ and $\mathrm{T}_{\mathrm{EM}}$ cells $(P<0.0001)$ in the blood of mice vaccinated via the IN route compared with the IP route at week 6 (Figure 3b) and week 16 (see Supplementary Figure S2). IP vaccination generated more $\mathrm{T}_{\mathrm{EFF}}, \mathrm{T}_{\mathrm{EM}}$, and KLRG1 + $\mathrm{T}_{\mathrm{EFF}}$ cells in the spleen compared with IN vaccination at week $1(P<0.01)$. At subsequent time points, however, there were significantly more $\mathrm{T}_{\mathrm{EFF}}$ and $\mathrm{T}_{\mathrm{EM}} \mathrm{M}$-specific CD8 $+\mathrm{T}$ cells in the spleen in the IN group compared with the IP group $(P<0.05)$. These data indicate that the route of vaccination influences CD8 + T-cell phenotype not only in the blood and tissue of the lung but also in the secondary lymphoid organs.

Inoculation into the lungs is necessary to generate lungtropic CD8 + T cells

Inoculation volume determines inoculum penetration into the lungs. ${ }^{34-36}$ A volume of $20 \mu \mathrm{l}$ will largely remain in the nose, $50 \mu \mathrm{l}$ will enter the upper respiratory tract, and $100 \mu \mathrm{l}$ will enter the lower respiratory tract. ${ }^{34}$ To determine whether inoculation into the lungs is necessary for the development of tissue-tropic $\mathrm{CD} 8+\mathrm{T}$ cells, we vaccinated mice IN with $1.2 \times 10^{5} \mathrm{PFU}$ of MCMV-M in a final volume of 20,50, or $100 \mu \mathrm{l}$ and determined the percentage of $\mathrm{M}$-specific $\mathrm{CD} 8+\mathrm{T}$ cells in the lung tissue and blood at week 6 post-vaccination. The tissue-tropic population of M-specific CD8 + T cells was highly dependent on inoculation volume (Figure 4a). Inoculation into the nose $(20 \mu \mathrm{l})$ was not sufficient to generate a response in the lung parenchyma, whereas progressively larger tissue-tropic $\mathrm{M}$-specific CD8 + T-cell populations were observed with inoculation volumes of 50 and $100 \mu \mathrm{l}$ (Figure 4a).

\section{IN vaccination leads to increased MCMV replication in the lung}

To test the hypothesis that vector replication is affected by the route of inoculation, we vaccinated mice with $100 \mu \mathrm{l}$ of MCMV$M$ via the IN or IP route. On days 2, 4, 8, 14, and 21 postvaccination, DNA was extracted from the lungs, salivary glands, and spleen, and viral loads were determined by quantitative PCR for the IE1 gene. ${ }^{37}$ Mice vaccinated IN had significantly higher MCMV genome copy numbers in the lungs at all time points (Figure $4 \mathbf{b} ; P<0.0001$ ). IP vaccination was associated with significantly higher viral loads in the spleen on day 2 , but no differences were detected on subsequent days. Viral replication in the salivary glands was identical in both vaccination groups. These data demonstrate that the route of administration affects the distribution and kinetics of MCMV replication. As there is greater MCMV replication in the lungs of mice infected via the IN route, it is likely that more antigen is presented at this site during acute infection and that a larger pool of latently MCMV-infected cells persists during chronic infection.

IN vaccination with MCMV-M leads to earlier antiviral T-cell responses after RSV challenge

Based on the dramatic differences in tissue localization and CD8 + T-cell memory phenotype, we hypothesized that mice vaccinated via the IN route would more readily control RSV 
a

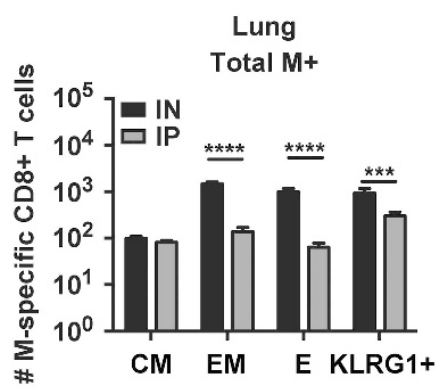

b

Week 6

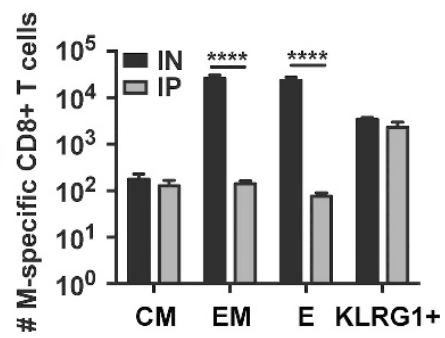

Lung

Tissue M+

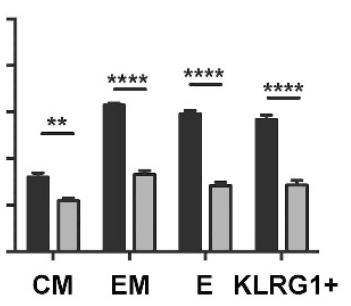

Lung

Blood M+

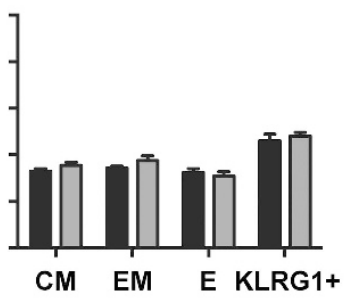

Spleen

White pulp M+

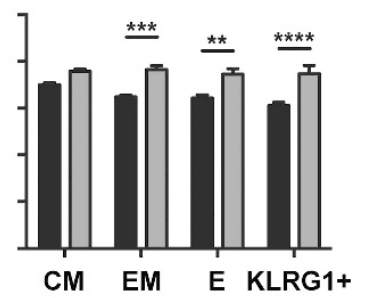

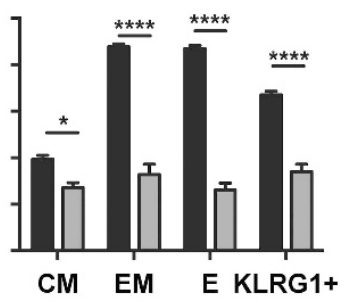
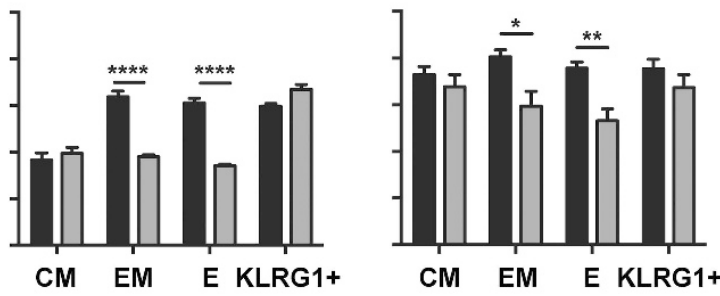

Figure 3 IN vaccination with MCMV-M augments the M-specific CD8 + T-cell response via the induction of effector and effector memory cells in the lung parenchyma and blood. The memory phenotype of M-specific CD8 + T cells harvested from the indicated sites was determined at week 1 and week 6 post-vaccination with MCMV-M. Mice were injected IV with anti-CD45 antibody 5 min prior to killing to identify cells in the blood or tissue. (a) Total number of central memory (CM), effector memory (EM), effector (E), and KLRG1 ${ }^{+}$effector (KLRG1 + ) cells at week 1 post-vaccination. (b) Total number of CM, EM, E, and KLRG1 + cells at week 6 post-vaccination. Bars represent mean \pm s.e.m. with five mice per group. ${ }^{* * *} P \leq 0.0001,{ }^{* * *} P<0.001,{ }^{* *} P<0.01$, and ${ }^{*} P<0.05$ by two-way analysis of variance with Tukey's post-test for multiple comparisons. Data represent two independent experiments. IN, intranasal; IP, intraperitoneal.

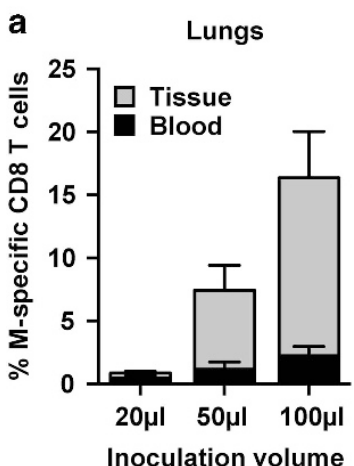

b

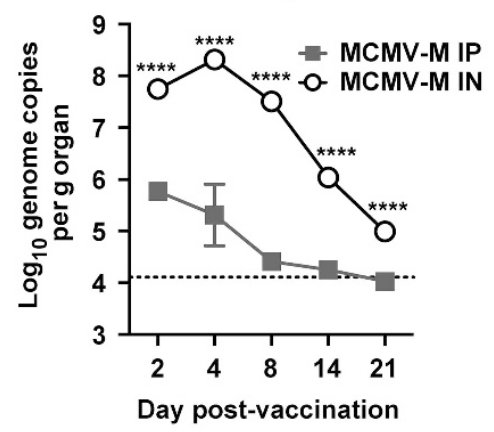

Spleen

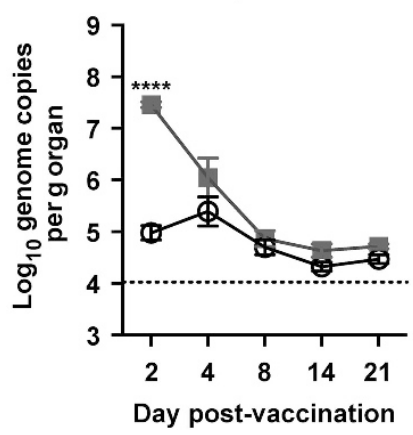

Salivary glands

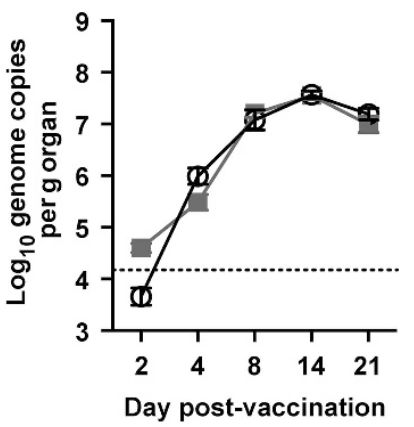

Figure 4 Large volume IN vaccination leads to increased viral replication in the lung and is necessary for the generation of tissue-tropic M-specific CD8 + T cells. (a) Mice were vaccinated IN with $1.2 \times 10^{5} \mathrm{PFU}$ of MCMV-M in a total volume of 20,50 , or $100 \mu$ l. The percentage of M-specific CD8 $+\mathrm{T}$ cells in the lung at 6 weeks post-vaccination was determined using tetramer staining and flow cytometry. Bars represent mean \pm s.e.m. with five mice per group. (b) Quantification of MCMV genome copy number by quantitative PCR in the lung, spleen, and salivary glands of mice infected IN or IP with $6 \times 10^{5}$ PFU of MCMV-M in $100 \mu \mathrm{l}$. Error bars represent s.e.m. Dotted line indicates limit of detection. ${ }^{* * *} P<0.0001$ by two-way analysis of variance with Tukey's post-test for multiple comparisons. IN, intranasal; IP, intraperitoneal; MCMV-M, murine cytomegalovirus vector expressing the respiratory syncytial virus matrix (M) protein.

infection. At week 16 post-vaccination with MCMV-M or an MCMV control, we challenged mice with RSV IN. On day 5 post-challenge, the lungs were harvested to measure viral load using a plaque assay. Mice infected with the control MCMV via either the IN or IP route had levels of RSV in their lungs similar to those detected in unvaccinated mice. In contrast, vaccination with MCMV-M via either route lead to decreased viral loads compared with controls $(P<0.0001$; Figure 5a). In addition, mice vaccinated IN with MCMV-M had RSV viral loads that were 100 -fold lower compared with mice vaccinated via the IP route $(P<0.001)$. To confirm the role of $\mathrm{CD} 8+\mathrm{T}$ cells in viral clearance, we injected MCMV-M-vaccinated mice with a depleting anti-CD8 antibody (2.43) on three consecutive days prior to RSV challenge. We confirmed depletion of CD8 $+\mathrm{T}$ cells in the blood and tissue of the lungs, spleen and the posterior mediastinal lymph node on the day of RSV challenge, and the CD8 + T-cell depletion was maintained through 5 days post-RSV challenge (see Supplementary Figure S3). When 


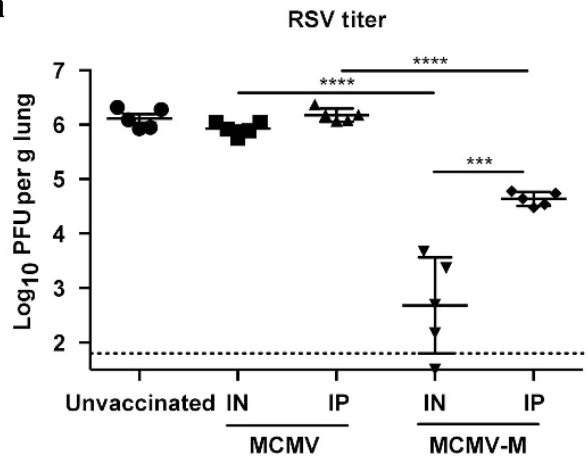

C

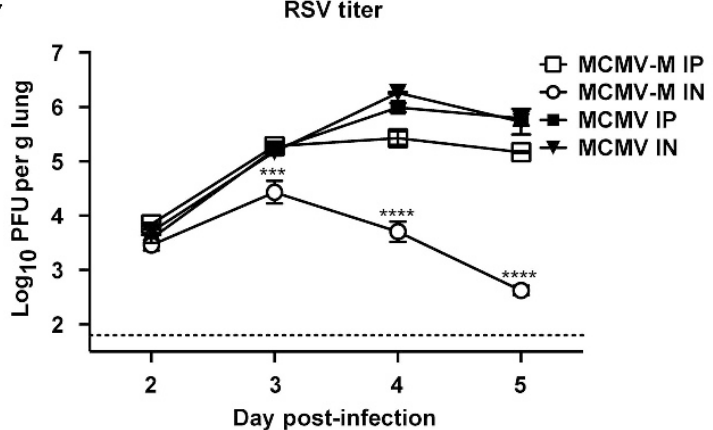

b

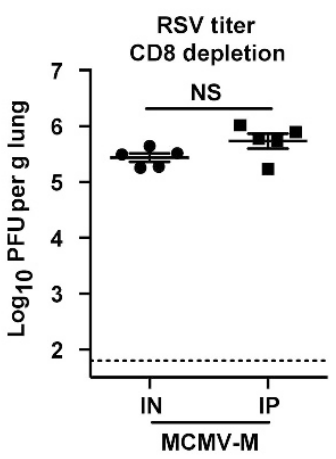

d

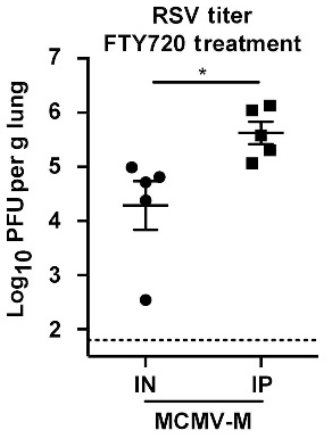

e

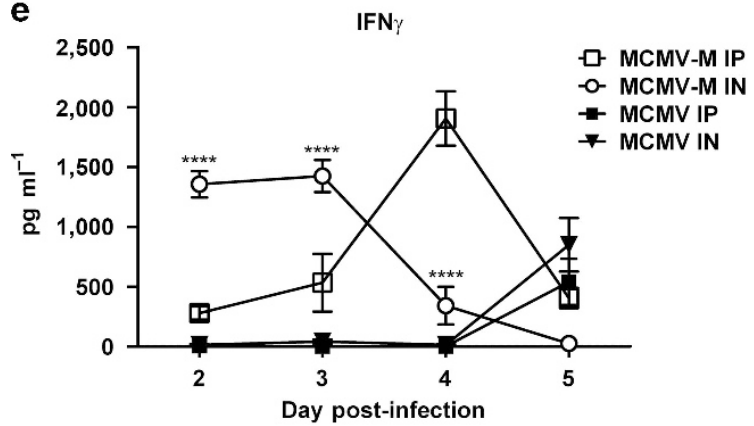

f

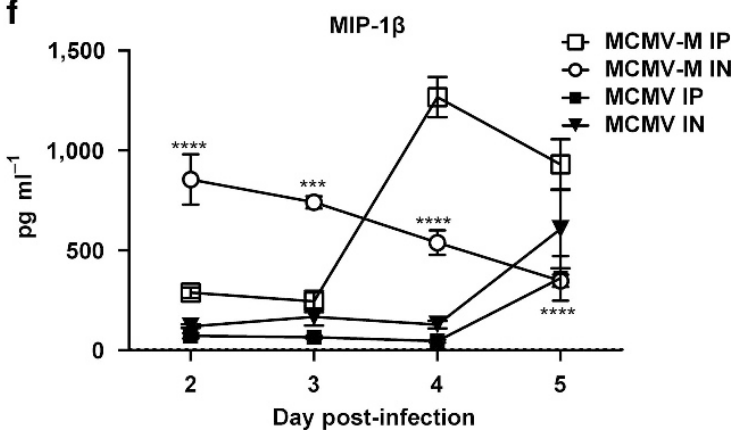

Figure 5 IN vaccination with MCMV-M leads to earlier antiviral responses compared to IP vaccination. Mice were challenged with $2 \times 10^{6} \mathrm{PFU}$ of RSV either in the absence of prior vaccination or 16 weeks after IN or IP administration of MCMV or MCMV-M. On days 2-5 post infection, the lungs were harvested for plaque assay and cytokine analysis. (a) Viral loads on day 5 post-infection determined by plaque assay. Dashed line indicates limit of detection. (b) Viral loads on day 5 post-infection after CD8 + T-cell depletion. Dashed line indicates limit of detection. NS = not significant. (c) Serial viral loads after challenge with RSV. Dashed line indicates limit of detection. Error bars indicate s.e.m. with 5 mice per group. Asterisks indicate significant differences between MCMV-M IN and MCMV-M IP. (d) Viral loads on day 5 post-infection after FTY720 treatment. Dashed line indicates limit of detection. $(\mathbf{e}, \mathbf{f})$ Concentration of interferon gamma (IFN $\gamma$ ) in e and macrophage inflammatory protein-1beta (MIP-1 $\beta$ ) in $f$ in the lungs determined by multiplex beadbased array after challenge with RSV. Asterisks indicate significant difference between MCMV-M IN and MCMV-M IP. ${ }^{* * \star \star} P<0.0001$, ${ }^{\star * \star} P<0.001$ and ${ }^{*} P<0.05$ by one-way analysis of variance with Tukey's post-test for multiple comparisons. IN, intranasal; IP, intraperitoneal; MCMV-M, murine cytomegalovirus vector expressing the respiratory syncytial virus matrix (M) protein; RSV, respiratory syncytial virus.

$\mathrm{CD} 8+\mathrm{T}$ cells were depleted, the difference in viral load between the IN and IP vaccination group was abrogated, indicating the $\mathrm{CD} 8+\mathrm{T}$-cell responses were responsible for the difference in viral loads between mice vaccinated IN and IP (Figure 5b).

To further support the role of $\mathrm{T}_{\mathrm{RM}}$ in mediating enhanced protection, we treated mice that had been vaccinated 16 weeks prior with MCMV-M by the IN or IP route with FTY720. FTY720 is a sphingosine-1-phospate receptor 1 agonist and prevents the egress of $\mathrm{T}$ cells from lymphoid organs. Treatment with FTY720 reduces the number of recirculating naïve and
$\mathrm{T}_{\mathrm{CM}}$ cells in the peripheral blood. ${ }^{38}$ Mice were injected IP with FTY720 daily for 3 days prior to RSV infection and throughout infection. FTY720 treatment significantly reduced the number of CD62L + M-specific CD8 + T cells in the blood of the lungs, but not in the tissue of mice vaccinated with MCMV-M by the IN or IP route $(P<0.05$; see Supplementary Figure S4b). When vaccinated mice were treated with FTY720 prior to RSV infection, mice vaccinated IN had significantly lower viral titers in lung than mice vaccinated IP, further supporting the critical role of $\mathrm{M}$-specific $\mathrm{CD} 8+\mathrm{T}_{\mathrm{RM}}$ cells in mediating viral control $(P<0.05$, Figure 5d). 
$\mathrm{CD} 8+\mathrm{T}_{\mathrm{RM}}$ cells have been shown to respond rapidly to antigen upon challenge. ${ }^{6} \mathrm{We}$ therefore hypothesized that IN vaccination, which generates more $\mathrm{T}_{\mathrm{RM}}$ cells, would lead to earlier antiviral responses. To evaluate early antiviral responses, we challenged mice vaccinated 16 weeks previously with MCMV-M or control MCMV. On day 2 through day 5 postchallenge, we harvested the lungs to determine viral load by plaque assay and cytokine levels by bead-based multiplex assay. Mice vaccinated with MCMV-M via the IN route had lower viral loads than mice vaccinated via the IP route at day 3 though day $5(P<0.001$; Figure $5 \mathbf{c})$. In addition, mice vaccinated with MCMV-M via the IP route had lower viral loads at day 4 and day 5 compared with mice vaccinated with control MCMV via either route $(P<0.01)$. However, viral replication relative to controls was suppressed more effectively $(P<0.0001)$ and more rapidly in mice vaccinated IN with MCMV-M. Peak RSV titers were 10 -fold lower in mice vaccinated with MCMV-M via the IN route compared with mice vaccinated via the IP route and nearly 100 -fold lower compared with mice vaccinated with control MCMV via the IN route $(P<0.0001)$. There were no significant differences in lung RSV titers at any time point after challenge between mice immunized IN or IP with control MCMV.

Next, we evaluated cytokine and chemokine levels in the lungs of vaccinated mice after RSV challenge. Mice vaccinated with MCMV-M via the IN route had significantly higher levels of interferon gamma and macrophage inflammatory proteinlbeta on day 2 and day 3 post-challenge compared with mice vaccinated via the IP route $(P<0.001$; Figure 5e, f). Cytokine and chemokine levels decreased by day 4 and day 5 as viral loads decreased. In mice vaccinated with MCMV-M via the IP route, peak levels of interferon gamma and macrophage inflammatory protein-1beta occurred on day 4 , one day later than IN vaccinated mice. Mice vaccinated with control MCMV did not show increased levels of interferon gamma and macrophage inflammatory protein-1beta until day 5 post-challenge with no difference between IN and IP vaccinated groups. These data suggest that the $T_{R M}$ population generated by IN vaccination with MCMV-M responds rapidly on challenge and mediates better control of RSV.

\section{DISCUSSION}

In this study, we show that IN administration of an MCMV vector expressing the M protein from RSV generates a robust and durable $\mathrm{M}$-specific $\mathrm{CD} 8+\mathrm{T}$-cell response. This mode of vaccine delivery drives the accumulation of $\mathrm{CD} 8+\mathrm{T}_{\mathrm{RM}}$ cells in the lung parenchyma, most likely as a consequence of greater antigen expression and viral replication at the site of inoculation. These $\mathrm{CD} 8+\mathrm{T}_{\mathrm{RM}}$ cells mediate early antiviral responses that rapidly control infection with RSV.

Here we show that local, rather than systemic delivery of a viral antigen, leads to the generation of tissue-resident CD8 + $\mathrm{T}$ cells in lung. Pulmonary inoculation has previously been shown to be necessary for the generation of $T_{R M}$ cells in the lung. ${ }^{39,40}$ Similar requirements have also been demonstrated for other mucosal tissues, including the intestine and female genital tract. ${ }^{41-43}$ Subcutaneous administration of rhCMV elicits CD8 $+\mathrm{T}$ cells in bronchoalveolar lavage samples in Rhesus macaques, suggesting that other routes of tissue inoculation may also induce $\mathrm{T}_{\mathrm{RM}}{ }^{44}$ IP vaccination with MCMV-M generated an M-specific CD8 + T-cell population that was primarily confined to the peripheral circulation, consistent with previously published data showing IP administration of MCMV generates a non-perfusable CD8 + T-cell population in the blood supply of the lungs, kidneys, and liver. ${ }^{45}$ Nonetheless, enhanced local immunity did not occur at the expense of systemic immunity, because equivalent numbers of $\mathrm{M}$-specific CD8 $+\mathrm{T}$ cells were located in the spleen after IN vaccination with MCMV-M. Interestingly, most of the $\mathrm{M}$-specific CD8 $+\mathrm{T}$ cells in the blood from both IN and IP vaccination do not express CD62L and were not depleted by FTY720 treatment, suggesting that this population does not recirculate, but rather remains resident in the marginating pool. This concept is consistent with a previous study using immune-chimera mice that demonstrated antigenspecific cells located in the vasculature of organs did not appear to recirculate. ${ }^{46}$

$\mathrm{T}_{\mathrm{RM}}$ cells are poised to respond quickly to antigen encounter and have been shown to act as immune sentinels via the rapid production of cytokines and chemokines. ${ }^{7,47}$ Consistent with these findings, we found that vaccination with MCMV-M via the IN route, which elicited robust populations of $\mathrm{T}_{\mathrm{RM}}$ cells, led to higher levels of interferon gamma and macrophage inflammatory protein-1beta in the lungs of mice at earlier time points post-challenge compared with mice vaccinated via the IP route. These enhanced immune responses mediated earlier clearance of RSV and lower peak viral titers, supporting an important role for $\mathrm{CD} 8+\mathrm{T}_{\mathrm{RM}}$ cells in pathogen control. Although our data demonstrate that CD8 $+\mathrm{T}$ cells play a major role in the increased protection seen with IN vaccination, the role of $\mathrm{CD} 4+\mathrm{T}$ cells in protection has not been elucidated. The RSV M protein also encodes a CD4 + T-cell epitope, and the contribution of $\mathrm{CD} 4+\mathrm{T}$ cells specific for this epitope is currently being evaluated. ${ }^{31}$

The route of inoculation affected not only the localization but also the memory phenotype of vaccine-induced CD $8+T$ cells. In mice vaccinated via the IP route, $\mathrm{M}$-specific $\mathrm{CD} 8+\mathrm{T}$ cells displayed a predominant KLRG1 $+\mathrm{T}_{\mathrm{EFF}}$ phenotype. These findings are consistent with previously published data showing that inflationary memory populations derive mainly from short-lived KLRG1 $+\mathrm{T}_{\mathrm{EFF}}$ cells that are constantly replenished. ${ }^{45}$ However, when we administer the same vector by the IN route, there is a significant shift in the phenotype of cells elicited to the same epitope. In mice vaccinated via the IN route, there were similar numbers of KLRG1 $+\mathrm{T}_{\mathrm{EFF}}$ and $\mathrm{T}_{\mathrm{CM}}$ cells, but also much larger populations of $\mathrm{T}_{\mathrm{EFF}}$ and $\mathrm{T}_{\mathrm{EM}}$ cells across all anatomical locations. These populations may possess a better capacity to respond to antigen re-exposure or longer lifespans since they do not express KLRG1. These data emphasize the critical role of the local environment of antigen-presentation in the character of the immune response. 
Steinert et al. ${ }^{46}$ recently demonstrated that CD8 + T-cell isolation from the tissue is biased by phenotype and location. As our data compare two different vaccination routes and all samples were treated identically, it is unlikely that our conclusions will be affected by such technological constraints although we may underestimate the number of $\mathrm{M}$-specific $\mathrm{CD} 8+\mathrm{T}$ cells. In addition, we were able to evaluate multiple parameters by flow cytometry, which allowed us to probe differences in memory phenotype that may have been missed by microscopy.

While most vaccination schedules require multiple doses or separate prime/boost constructs to minimize anti-vector immunity, CMV vectors encoding inflationary epitopes may require only a single dose to generate robust, durable protection. In particular, $\mathrm{T}_{\mathrm{RM}}$ populations in the lung have been shown to wane over time. ${ }^{39,48}$ In contrast, IN vaccination with a single dose of MCMV-M generated a long-lived $\mathrm{T}_{\mathrm{RM}}$ population, potentially overcoming the problem of waning immunity in the lung. $\mathrm{CD} 8+\mathrm{T}$ cells elicited by $\mathrm{CMV}$ infection do not assume an exhausted phenotype, but instead remain functional and protect in vivo. ${ }^{25,26,49}$ Moreover, persistent antigen expression leads to the generation of CD8 $+\mathrm{T}_{\mathrm{EFF}}$ and $\mathrm{T}_{\mathrm{EM}}$ cells, which respond more rapidly compared with the $\mathrm{T}_{\mathrm{CM}}$ cells typically generated by acute vectors.

Collectively, we demonstrate that local delivery of a persistent MCMV vector results in a high magnitude of $\mathrm{CD} 8+$ effector $\mathrm{T}_{\mathrm{RM}}$ cells with a single dose leading to early viral control and reduced viral loads. Sustained $\mathrm{T}_{\mathrm{RM}}$ populations will likely be a key feature of vaccines that protect against infectious diseases that are dependent on T-cell-mediated clearance for protection such as tuberculosis, malaria, and HIV.

\section{METHODS}

Mice. Age-matched (6-10 weeks) female CB6F1/J mice (Jackson Laboratories, Bar Harbor, ME) were used in all experiments. Mice were housed in the animal care facility at the National Institute of Allergy and Infectious Diseases under specific-pathogen-free conditions and maintained on standard rodent chow and water supplied ad libitum. All studies were reviewed and approved by the National Institutes of Health Animal Care and Use Committee.

Cell lines. Mouse embryonic fibroblasts (SCRC-1008) cell lines were obtained from the ATCC and cultured in Advanced Dulbecco's modified Eagle's medium (Invitrogen, Grand Island, NY) containing $8 \%$ or $10 \%$ fetal bovine serum or neonatal calf serum, 2 mm glutamine, $10 \mathrm{U} \mathrm{ml}^{-1}$ penicillin, $10 \mu \mathrm{g} \mathrm{ml}^{-1}$ streptomycin and $0.1 \mathrm{M}$ HEPES. HEp-2 cells were grown in Eagle's minimal essential medium supplemented with $10 \%$ fetal bovine serum, $2 \mathrm{~mm}$ glutamine, $10 \mathrm{U} \mathrm{ml}^{-1}$ penicillin, $10 \mu \mathrm{g} \mathrm{ml}^{-1}$ streptomycin and $0.1 \mathrm{M}$ HEPES.

Viruses and infections. Recombinant MCMVs were made using a bacterial artificial chromosome (BAC) system. ${ }^{21}$ The RSV M sequence was inserted into the IE2 gene of the K181 $\Delta \mathrm{m} 157$ strain of MCMV by two-step allele replacement. BACs were extracted from Escherichia coli using a NucleoBond Xtra Maxi Prep Kit (Macherey-Nagel, Bethlehem, PA). Mouse embryonic fibroblasts cells were transfected with recombinant BACs via calcium phosphate precipitation (Clontech, Mountain View, CA). Viruses were passaged and single plaques isolated by serial dilution. Single plaques were selected based on excision of the BAC cassette by loss of GFP, and the presence of the RSV M gene was confirmed by PCR. MCMV stocks were produced by sonication of infected mouse embryonic fibroblasts cells. Mice were infected IN or IP with $6 \times 10^{5} \mathrm{PFU}$ of recombinant MCMV-M viruses in $100 \mu \mathrm{l}$ of $10 \%$ fetal bovine serum Dulbecco's modified Eagle's medium, unless otherwise noted. RSV stocks for challenge were generated from the A2 strain of RSV by sonication of infected HEp-2 monolayers. ${ }^{34}$ Mice were challenged IN with $2 \times 10^{6} \mathrm{PFU}$ of RSV in $100 \mu \mathrm{l}$ of $10 \%$ minimum essential media. All IN inoculations were performed following anesthesia with isoflourane (3\%). Mice were killed by lethal overdose with pentobarbital $\left(250 \mathrm{mg} \mathrm{kg}^{-1}\right)$.

Intravascular staining and flow cytometry. For intravascular staining, mice were injected intravenously with $3 \mu \mathrm{g}$ of anti-CD45 antibody (BD Biosciences, San Jose, CA). Lungs and spleen were harvested after euthanasia with pentobarbital $\left(250 \mathrm{mg} \mathrm{kg}^{-1}\right)$. Lymphocytes were isolated by physical disruption of tissue using a GentleMACs machine (Miltenyi Biotec, San Diego, CA) followed by Fico-LITE density gradient centrifugation. Isolated mononuclear cells were washed with phosphate-buffered saline and resuspended in FACS staining buffer (phosphate-buffered saline $+1 \%$ fetal bovine serum $+0.05 \%$ sodium azide). Cells were stained with fluorochrome-labeled antibodies for the lineage markers CD3 (145-2C11) and CD8 (53-6.7) and the phenotypic markers CD44 (IM7), CD62L (MEL-14), CD127 (A7R34), KLRG-1 (2F1/KLRG1), CD69 (H1.2F3), and CD103 (M290) (BD Biosciences or BioLegend, San Diego, CA). The amine viability dye AquaBlue (Invitrogen) was used to identify dead cells. Antigen specificity was determined using $\mathrm{D}^{\mathrm{b}} \mathrm{M}_{187-195}$ tetramers conjugated to APC (MBL, Woburn, MA). All samples were stained with a pre-mixed antibody cocktail for $20 \mathrm{~min}$ at $4{ }^{\circ} \mathrm{C}$. Data were collected using an LSR II flow cytometer (BD Biosciences) and analyzed with FlowJo software (TreeStar, San Carlos, CA).

RSV plaque assay and cytokine analysis. Plaque assays were performed as described previously. ${ }^{50}$ Lungs were harvested, weighed, and frozen quickly in $10 \%$ minimum essential media supplemented with $2 \mathrm{~mm}$ glutamine, $10 \mathrm{U} \mathrm{ml}^{-1}$ penicillin, and $10 \mu \mathrm{g} \mathrm{ml}^{-1}$ streptomycin. After thawing, lung tissue was dissociated using the GentleMACs machine on program lung_02 (Miltenyi). Cell suspensions were pelleted to remove cellular debris. Supernatants were serially diluted and inoculated on $80 \%$ confluent HEp-2 cell monolayers in triplicate. Cells were incubated for $1 \mathrm{~h}$ at room temperature and overlaid with $1 \%$ methyl cellulose in $10 \%$ minimum essential media. After 4 days at $37^{\circ} \mathrm{C}$, cells were fixed with $10 \%$ buffered formalin and stained with hematoxylin and eosin. Plaques were counted and expressed as $\log _{10}$ PFU per g of lung tissue. The limit of detection was 1.8 $\log _{10}$ PFU per g. For cytokine analysis by multiplex bead-based array, supernatants were sent to AssayGate (Ijamsville, MD).

CD8 + T-cell depletion and FTY720 treatment. Mice were vaccinated with MCMV-M via the IN or IP route. After 16 weeks, $200 \mu \mathrm{g}$ of anti-CD8 antibody (clone 4.23) in a total volume of $200 \mu \mathrm{l}$ was injected IP for three consecutive days for CD8 + T-cell depletion studies. On the third day, mice were challenged IN with $2 \times 10^{6} \mathrm{PFU}$ of RSV. Five days after challenge, the left lobe of the lung was harvested for plaque assay. For FTY720 experiments, mice that had been vaccinated with MCMV-M via the IN or IP route 16 weeks prior were given daily IP injections of FTY720 at a dose of $1 \mathrm{mg} \mathrm{kg}^{-1}$ (Sigma-Aldrich, St Louis, MO) beginning 3 days prior RSV infection until killed. On the fourth day of FTY720 treatment, mice were challenged with RSV as described above.

Real-time quantitative PCR. Samples were processed as described above for the plaque assay. DNA was extracted from $200 \mu \mathrm{l}$ of supernatant using a Nucleospin Blood Kit (Clontech). Real-time quantitative PCR was performed for the MCMV IE1 gene as described previously. ${ }^{37}$

Statistical analysis. Statistical analyses were performed using one-way or two-way analysis of variances in GraphPad Prism (La Jolla, CA). 
SUPPLEMENTARY MATERIAL is linked to the online version of the paper at http://www.nature.com/mi

\section{ACKNOWLEDGMENTS}

We thank Brenda Hartman for help with figure preparation. This work was supported by intramural funding from the Vaccine Research Center, National Institute of Allergy and Infectious Diseases, National Institutes of Health. DAP is a Wellcome Trust Senior Investigator.

\section{DISCLOSURE}

The authors declare no conflicts of interest.

Official journal of the Society for Mucosal Immunology

\section{REFERENCES}

1. Shane, H.L. \& Klonowski, K.D. Every breath you take: the impact of environment on resident memory CD8 Tcells in the lung. Front. Immunol. 5, 320 (2014)

2. Masopust, D., Vezys, V., Marzo, A.L. \& Lefrancois, L. Preferential localization of effector memory cells in nonlymphoid tissue. Science 291, 2413-2417 (2001).

3. Gebhardt, T., Wakim, L.M., Eidsmo, L., Reading, P.C., Heath, W.R. \& Carbone, F.R. Memory Tcells in nonlymphoid tissue that provide enhanced local immunity during infection with herpes simplex virus. Nat. Immunol. 10, 524-530 (2009).

4. Ariotti, S. et al. Tcell memory. Skin-resident memory CD8(+) Tcells trigger a state of tissue-wide pathogen alert. Science 346, 101-105 (2014).

5. Schenkel, J.M. \& Masopust, D. Tissue-resident memory T cells. Immunity 41, 886-897 (2014).

6. Schenkel, J.M., Fraser, K.A., Beura, L.K., Pauken, K.E., Vezys, V. \& Masopust, D. Tcell memory. Resident memory CD8 Tcells trigger protective innate and adaptive immune responses. Science 346, 98-101 (2014).

7. McMaster, S.R., Wilson, J.J., Wang, H. \& Kohlmeier, J.E. Airway-resident memory CD8 Tcells provide antigen-specific protection against respiratory virus challenge through rapid IFN-gamma production. J. Immunol. 195, 203-209 (2015).

8. Jiang, X., Clark, R.A., Liu, L., Wagers, A.J., Fuhlbrigge, R.C. \& Kupper, T.S. Skin infection generates non-migratory memory CD8 $+\mathrm{T}(\mathrm{RM})$ cells providing global skin immunity. Nature 483, 227-231 (2012).

9. Robinson, H.L. \& Amara, R.R. T cell vaccines for microbial infections. Nat. Med. 11, S25-S32 (2005).

10. Wherry, E.J. \& Ahmed, R. Memory CD8 T-cell differentiation during viral infection. J. Virol. 78, 5535-5545 (2004).

11. Sallusto, F., Geginat, J. \& Lanzavecchia, A. Central memory and effector memory Tcell subsets: function, generation, and maintenance. Annu. Rev. Immunol. 22, 745-763 (2004).

12. Hansen, S.G. et al. Profound early control of highly pathogenic SIV by an effector memory T-cell vaccine. Nature 473, 523-527 (2011).

13. Hansen, S.G. et al. Immune clearance of highly pathogenic SIV infection. Nature 502, 100-104 (2013).

14. Tierney, R. et al. A single-dose cytomegalovirus-based vaccine encoding tetanus toxin fragment $\mathrm{C}$ induces sustained levels of protective tetanus toxin antibodies in mice. Vaccine 30, 3047-3052 (2012).

15. Tsuda, Y. et al. A cytomegalovirus-based vaccine provides long-lasting protection against lethal Ebola virus challenge after a single dose. Vaccine 33, 2261-2266 (2015).

16. Wang, X. et al. Murine cytomegalovirus abortively infects human dendritic cells, leading to expression and presentation of virally vectored genes. J. Virol. 77, 7182-7192 (2003).

17. Beverley, P.C. et al. A novel murine cytomegalovirus vaccine vector protects against Mycobacterium tuberculosis. J. Immunol. 193, 2306-2316 (2014).

18. Tsuda, Y. et al. A replicating cytomegalovirus-based vaccine encoding a single Ebola virus nucleoprotein CTL epitope confers protection against Ebola virus. PLoS Negl. Trop. Dis. 5, e1275 (2011).

19. Klyushnenkova, E.N. et al. A cytomegalovirus-based vaccine expressing a single tumor-specific CD8 + T-cell epitope delays tumor growth in a murine model of prostate cancer. J. Immunother. 35, 390-399 (2012).
20. Xu, G., Smith, T., Grey, F. \& Hill, A.B. Cytomegalovirus-based cancer vaccines expressing TRP2 induce rejection of melanoma in mice. Biochem. Biophys. Res. Commun. 437, 287-291 (2013).

21. Redwood, A.J. et al. Use of a murine cytomegalovirus K181-derived bacterial artificial chromosome as a vaccine vector for immunocontraception. J. Virol. 79, 2998-3008 (2005).

22. Karrer, $U$. et al. Memory inflation: continuous accumulation of antiviral CD8 + T cells over time. J. Immunol. 170, 2022-2029 (2003).

23. Sierro, S., Rothkopf, R. \& Klenerman, P. Evolution of diverse antiviral CD8 + T cell populations after murine cytomegalovirus infection. Eur. J. Immunol. 35, 1113-1123 (2005).

24. Sylwester, A.W. et al. Broadly targeted human cytomegalovirus-specific $\mathrm{CD} 4+$ and $\mathrm{CD} 8+\mathrm{T}$ cells dominate the memory compartments of exposed subjects. J. Exp. Med. 202, 673-685 (2005).

25. Holtappels, R., Thomas, D., Podlech, J. \& Reddehase, M.J. Two antigenic peptides from genes $\mathrm{m} 123$ and $\mathrm{m} 164$ of murine cytomegalovirus quantitatively dominate CD8 T-cell memory in the $\mathrm{H}-2 \mathrm{~d}$ haplotype. J. Virol. 76, 151-164 (2002).

26. Karrer, U. et al. Expansion of protective CD8 + T-cell responses driven by recombinant cytomegaloviruses. J. Virol. 78, 2255-2264 (2004).

27. Munks, M.W., Cho, K.S., Pinto, A.K., Sierro, S., Klenerman, P. \& Hill, A.B. Four distinct patterns of memory CD8 T cell responses to chronic murine cytomegalovirus infection. J. Immunol. 177, 450-458 (2006).

28. Appay, $V$. et al. Memory $\mathrm{CD} 8+T$ cells vary in differentiation phenotype in different persistent virus infections. Nat. Med. 8, 379-385 (2002).

29. Holtappels, R., Pahl-Seibert, M.F., Thomas, D. \& Reddehase, M.J. Enrichment of immediate-early 1 (m123/pp89) peptide-specific CD8 T cells in a pulmonary CD62L(Io) memory-effector cell pool during latent murine cytomegalovirus infection of the lungs. J. Virol. 74, 11495-11503 (2000).

30. Snyder, C.M., Cho, K.S., Bonnett, E.L., van Dommelen, S., Shellam, G.R. \& Hill, A.B. Memory inflation during chronic viral infection is maintained by continuous production of short-lived, functional T cells. Immunity $\mathbf{2 9}$, 650-659 (2008).

31. Liu, J., Ruckwardt, T.J., Chen, M., Johnson, T.R. \& Graham, B.S. Characterization of respiratory syncytial virus $\mathrm{M}$ - and M2-specific CD4 T cells in a murine model. J. Virol. 83, 4934-4941 (2009).

32. Liu, J. et al. A Numerically subdominant CD8 T cell response to matrix protein of respiratory syncytial virus controls infection with limited immunopathology. PLoS Pathog. 12, e1005486 (2016).

33. Anderson, K.G. et al. Intravascular staining for discrimination of vascular and tissue leukocytes. Nat. Protoc. 9, 209-222 (2014).

34. Graham, B.S., Perkins, M.D., Wright, P.F. \& Karzon, D.T. Primary respiratory syncytial virus infection in mice. J. Med. Virol. 26, 153-162 (1988).

35. Southam, D.S., Dolovich, M., O’Byrne, P.M. \& Inman, M.D. Distribution of intranasal instillations in mice: effects of volume, time, body position, and anesthesia. Am. J. Physiol. Lung Cell. Mol. Physiol. 282, L833-L839 (2002).

36. Miller, M.A. et al. Visualization of murine intranasal dosing efficiency using luminescent Francisella tularensis: effect of instillation volume and form of anesthesia. PLoS One 7, e31359 (2012).

37. Tang-Feldman, Y.J., Wojtowicz, A., Lochhead, G.R., Hale, M.A., Li, Y. \& Pomeroy, C. Use of quantitative real-time PCR (qRT-PCR) to measure cytokine transcription and viral load in murine cytomegalovirus infection. J. Virol. Methods 131, 122-129 (2006).

38. Hofmann, M., Brinkmann, V. \& Zerwes, H.G. FTY720 preferentially depletes naive $\mathrm{T}$ cells from peripheral and lymphoid organs. Int. Immunopharmacol. 6, 1902-1910 (2006).

39. Wu, T. et al. Lung-resident memory CD8 Tcells (TRM) are indispensable for optimal cross-protection against pulmonary virus infection. J. Leukoc. Biol. 95, 215-224 (2014).

40. Anderson, K.G. et al. Cutting edge: intravascular staining redefines lung CD8 T cell responses. J. Immunol. 189, 2702-2706 (2012).

41. Cuburu, N. et al. Intravaginal immunization with HPV vectors induces tissue-resident CD8 + T cell responses. J. Clin. Invest. 122, 4606-4620 (2012). 
42. Gallichan, W.S. \& Rosenthal, K.L. Long-lived cytotoxic T lymphocyte memory in mucosal tissues after mucosal but not systemic immunization. J. Exp. Med. 184, 1879-1890 (1996).

43. Belyakov, I.M. et al. Mucosal immunization with HIV-1 peptide vaccine induces mucosal and systemic cytotoxic $T$ lymphocytes and protective immunity in mice against intrarectal recombinant HIV-vaccinia challenge. Proc. Natl. Acad. Sci. USA 95, 1709-1714 (1998).

44. Hansen, S.G. et al. Effector memory T cell responses are associated with protection of rhesus monkeys from mucosal simian immunodeficiency virus challenge. Nat. Med. 15, 293-299 (2009).

45. Smith, C.J., Turula, H. \& Snyder, C.M. Systemic hematogenous maintenance of memory inflation by MCMV infection. PLOS Pathog. 10, e1004233 (2014).

46. Steinert, EM et al. Quantifying memory CD8 T cells reveals regionalization of immunosurveillance. Cell 161, 737-749 (2015).

47. Schenkel, J.M., Fraser, K.A., Vezys, V. \& Masopust, D. Sensing and alarm function of resident memory CD8(+) T cells. Nat. Immunol. 14, 509-513 (2013).

48. Anderson, K.G. \& Masopust, D. Editorial: pulmonary resident memory CD8 T cells: here today, gone tomorrow. J. Leukoc. Biol. 95, 199-201 (2014).
49. Pahl-Seibert, M.F. et al. Highly protective in vivo function of cytomegalovirus IE1 epitope-specific memory CD8 T cells purified by T-cell receptorbased cell sorting. J. Virol. 79, 5400-5413 (2005).

50. Ruckwardt, T.J. et al. Neonatal CD8 T-cell hierarchy is distinct from adults and is influenced by intrinsic T cell properties in respiratory syncytial virus infected mice. PLoS Pathog. 7, e1002377 (2011).

(1) (2) This work is licensed under a Creative Commons Attribution-NonCommercial-ShareAlike $\quad 4.0$ International License. The images or other third party material in this article are included in the article's Creative Commons license, unlessindicated otherwise in the creditline; if the material is not included under the Creative Commons license, users will need to obtain permission from the license holder to reproduce the material. To view a copy of this license, visit http:// creativecommons.org/licenses/by-nc-sa/4.0/

(C) The Author(s) 2017 